Check for updates

Cite this: RSC Adv., 2019, 9, 35384

\title{
Highly transparent copper iodide thin film thermoelectric generator on a flexible substrate
}

\author{
J. Coroa, (D) ${ }^{a}$ B. M. Morais Faustino, (D) *a A. Marques, ${ }^{a}$ C. Bianchi, ${ }^{a}$ T. Koskinen, (D) \\ T. Juntunen, $\mathbb{D}^{\mathrm{b}}$ I. Tittonen ${ }^{\mathrm{b}}$ and I. Ferreira ${ }^{\mathrm{a}}$
}

Simultaneously transparent and flexible conductive materials are in demand to follow the current trend in flexible technology. The search for materials with compliant optoelectronic properties, while simultaneously retaining their electric conductivity at high strain deformation, comprises promising opportunities in modern nanotechnology. Copper iodide (Cul) is not only the most transparent and highly conductive $p$-type material, but its optimization has contributed to improved $Z T$ values in planar thin-film thermoelectrics. In this work, the readiness of Cul thin films to transparent, flexible technology is evidenced. A maximum ZT value of 0.29 for single Cul thin films of ca. $300 \mathrm{~nm}$ in thickness is reported. Values of open-circuit voltage $V_{o c}$ short circuit current $I_{s c}$ and power output of $p-n$ thermoelectric modules of Gallium-doped zinc oxide (GZO) and Cul thin films deposited on a transparent flexible Kapton ${ }^{\circledR}$ (type CS) substrate are reported, and a prototype of a flexible transparent thermoelectric generator based on $17 \mathrm{p}-\mathrm{n}$ modules was constructed. Bending analysis of Cul thin films reveals interesting, distinct results when submitted to compression and tension analysis - a behaviour not seen in conventional semiconducting thin films under equivalent strain conditions. A plausible account for such diversity is also included.

Received 11th September 2019 Accepted 23rd October 2019

DOI: $10.1039 / c 9 r a 07309 d$

rsc.li/rsc-advances efficient process towards the production of planar, transparent TE thin films with $Z T$ values of up to $0.22 .^{5}$ This significant improvement maximises the TE efficiency of wide-gap nonoxide p-type materials. Thermoelectric performance largely depends on the electronic composition near the valence band maximum of any material. The fact that a maximum Seebeck coefficient of $250 \mu \mathrm{V} \mathrm{K}^{-1}$ and a hole concentration up to $10^{20}$ $\mathrm{cm}^{-3}$ can be theoretically predicted based on first-principle calculations, ${ }^{6}$ means that currently studies no longer focus on the novelty of the CuI material, but rather on applying it to current technology. Whether applied to rectification layers in solar cells ${ }^{7}$ or in $\mathrm{p}-\mathrm{n}$ thermoelectric modules, ${ }^{5}$ the current trend in transparent technology is towards materials that are simultaneously transparent in the visible range of the spectrum and offer some degree of bending flexibility. ${ }^{\mathbf{8} 9}$ In this study, the first highly transparent and flexible $\mathrm{p}-\mathrm{n}$ thermoelectric generator (TEG) of $17 \mathrm{p}-\mathrm{n}$ modules electrically connected in series and thermally in parallel was successfully constructed and tested at temperature gradients of up to $30{ }^{\circ} \mathrm{C}$.

\section{Experimental}

Copper thin films were fabricated by resistive thermal evaporation under vacuum at a rate of $0.1 \mathrm{~nm} \mathrm{~s}^{-1}$ to reach an average $\mathrm{Cu}$ thickness of $60 \mathrm{~nm}$ onto Kapton (type CS) flexible substrates of $50 \mu \mathrm{m}$ thick - a polyimide transparent substrate as supplied by Dupont ${ }^{\mathrm{TM}}$. 
Afterwards, the iodination of $\mathrm{Cu}$ thin films was performed according to optimal conditions determined in a previous study. ${ }^{5}$ The iodination occurred in a sealed box purged with $\mathrm{N}_{2}$ prior to and post the addition of solid iodine (purity 99.9\% supplied by Sigma Aldrich®) on top of $\mathrm{Cu}$ thin films for a period of $60 \mathrm{~min}$ to yield a transparent $\gamma$-CuI thin film:

$$
2 \mathrm{Cu}+\mathrm{I}_{2(\mathrm{~s})} \rightarrow 2 \mathrm{CuI}
$$

To maximise the TEG power output, several $\mathrm{p}$ - and $\mathrm{n}$ - TE modules were electrically connected in series and thermally in parallel through transparent indium tin oxide (ITO) conductive electrodes. The TE modules were fabricated with masks applied to a $10 \times 10 \mathrm{~cm}^{2}$ transparent flexible Kapton ${ }^{\circledR}$ substrate, used as received from DuPont ${ }^{\mathrm{TM}}$. The process required the use of 2 masks with multiple $1 \times 1 \mathrm{~cm}^{2}$ square holes, carefully designed to allow the alternating deposition of $\mathrm{p}-\mathrm{n}$ elements. Gallium doped zinc oxide ( $c a .300 \mathrm{~nm}$ thick) was chosen as the n-type counterpart and this was deposited by RF magnetron sputtering using a GZO (ZnO : Ga $95: 5$ ) target supplied by Plasmaterials for a period of 60 min using an RF power in the range of $100-150 \mathrm{~W}$ and a working pressure between 1.2-2.0 mTorr. Conductive electrodes of indium tin oxide (ITO, ca. $300 \mathrm{~nm}$ thick) were also sputtered and provided $80 \Omega$. The ITO sputtering targets were supplied by Super Conductor Materials Inc.

TEG bending properties and stability were studied over repeated bending cycles using a home-build setup (Fig. 1) with in situ resistance measurement as a function of the bending radius. These profiles acquired to each cycle were performed according to a widely referenced method ${ }^{\mathbf{1 0}}$ which delivered the calculus of the critical bending radius for each cycle. This is defined as the bending curvature radius at which the resistance exceeds $5 \%$ or $10 \%$ of its original value. Generally, the setup consists of two grips, whereas one is attached to a non-movable arm holding the sample at one end of the substrate, and the other one is attached at a stage that moves back and forth with an accuracy and minimal step increment of $\sim 0.01 \mathrm{~mm}$. Fig. 1 shows the substrate at the initial length $L$, bent to an end-to-end length of $L-\mathrm{d} L$ and the bending radius $r$ at the centre of the sample, computed using eqn (1). The initial length of the sample and rate of change of the length are denoted by $L$ and $\mathrm{d} L / L$, and $h$ is the sum of the substrate thickness, $t_{\mathrm{s}}$, and the film thickness, $t_{\mathrm{f}}$.

$$
r=\frac{L}{2 \pi \sqrt{\frac{\mathrm{d} L}{L}-\frac{\pi^{2} h^{2}}{12 L^{2}}}}
$$

\section{Results and discussion}

Optoelectronic and thermoelectric properties were investigated in $\mathrm{CuI}$ and GZO elements individually prior to their application into $\mathrm{p}-\mathrm{n}$ thermoelectric modules. The properties of $\mathrm{CuI}$ and GZO films deposited on flexible Kapton CS® substrates were also tested with respect to changes in electrical conductivities during bending.

\subsection{Transparency of CuI and GZO}

Copper iodide is a wide band semiconductor with very high transmittance in the visible range of the spectrum $(>70 \%)$ and with significant absorption at the direct band gap (ca. $3 \mathrm{eV}$ ), where free excitons recombine, and copper vacancy defects dominantly contribute to the band transport. A characteristic split-off band is seen at $0.6 \mathrm{eV}$ above the valence band. Such an acceptor level enhances mobility by providing electron-hole delocalization at the top of the valence band. ${ }^{11}$ Similarly, gallium-doped zinc oxide thin films are extremely transparent in the visible range ( $>80 \%$ transmittance) owing to its wide-gap. Fig. 2 reveals the transmittance of both GZO and $\mathrm{CuI}$ thin film on Kapton CS substrate using UV-VIS absorption spectroscopy.

\subsection{Thermoelectric properties of $\mathrm{CuI}$ and GZO}

Table 1 summarizes the thermoelectric efficiency of both p-type $\mathrm{CuI}$ and n-type GZO films. The effect of the iodination process and iodine exposure time on the electrical conductivity of $\mathrm{CuI}$ has been extensively investigated in our previous study. ${ }^{5}$ Note that, although the Seebeck coefficient of GZO is significantly lower than that of CuI, its electrical conductivity is of one order of magnitude higher than that of CuI, which leads to similar power factors. The thermal conductivity of CuI and GZO are 0.48 and $2.17 \mathrm{~W}\left(\mathrm{~m}^{2} \mathrm{~K}\right)^{-1}$, respectively. As a result, the $Z T$ values of CuI and GZO are 0.29 and 0.07 , respectively.

\subsection{The flexibility of $\gamma$-CuI and GZO on Kapton CS}

The bending flexibility of each semiconducting film deposited on Kapton CS substrate was separately characterized through compressive and tensile strength analysis. As illustrated in
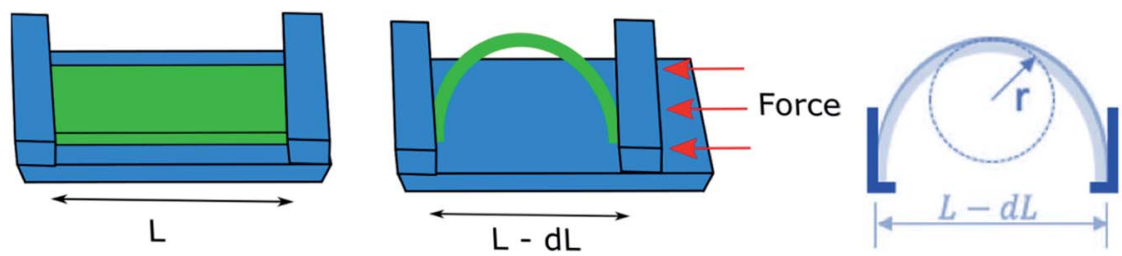

Fig. 1 Schematics of the home made bending machine and the consequent bending radius $r$ determined for each displacement $L-\mathrm{d} L$. The resistance of the sample is measured through two conductive wires that are connected to the sample ends. 

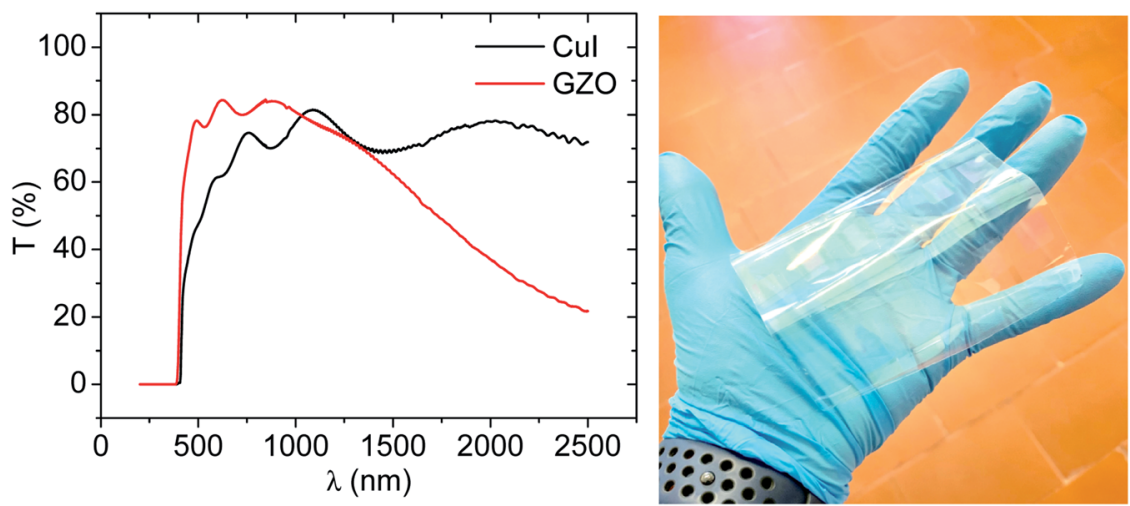

Fig. 2 Transmittance spectra of Cul and GZO thin films on Kapton CS® substrate (Left); A $10 \times 10 \mathrm{~cm}^{2}$ Kapton based prototype with alternating $\mathrm{p}-\mathrm{n}$ type modules of $\mathrm{Cul}$ and GZO, interconnected electrically in series with ITO.

Fig. 3, the type of force applied to the thin film (compressive or tension) depends on which side of the substrate the film is deposited on. Along with the critical bending radius, that is a direct measure of the maximum radius of the bending curvature before irreversible film destruction, Fig. 4 demonstrates that the type of force applied to the film during bending is also critical in defining electrical stability.

A complete bending cycle involves the bending and releasing of the sample, where values of the initial $\left(R_{0}\right)$ and final $\left(R_{\mathrm{f}}\right)$ resistance are noted after each cycle. As previously described, the critical bending radius is defined as the curvature at which the internal film resistance $\left(R_{\text {int }}\right)$ exceeds $5 \%$ or $10 \%$ of its original value. As such, it is only reasonable to comment on values of critical bending radius for samples exposed to tension forces. In this case, after the first bending cycle under tension, $\mathrm{CuI}$ has kept a resistance variation below $10 \%$ but with a critical bending radius of $15.5 \mathrm{~mm}$ assessed at values of film resistance exceeding $5 \%$ of $R_{0}$. During compression mode, the resistance variation was always below $5 \%$. Hence, in subsequent cycling tests, aiming at assessing the semiconducting films performance reliability under continuous bending, the bending radius was varied out of the critical range to values of up to 18.6 $\mathrm{mm}$. Fig. 5A shows that over 100 cycles, the compressive force applied is producing an electrical resistance variation of less than $5 \%$ of the initial resistance of the film. However, Fig. 5B shows that CuI thin films subjected to a tensile force result in the electrical resistance being linearly degraded with the number of bending cycles up to 80 cycles, beyond which a plateau of change in $R$ seems to be established. A total of 100 bending cycles produces an average variation of $9 \%$ of the initial resistance $R_{0}$. However, as also shown in Fig. $5 \mathrm{~B}$, when $\mathrm{CuI}$ is subjected to compressive forces, a maximum of $3.6 \%$ of $R_{0}$ is
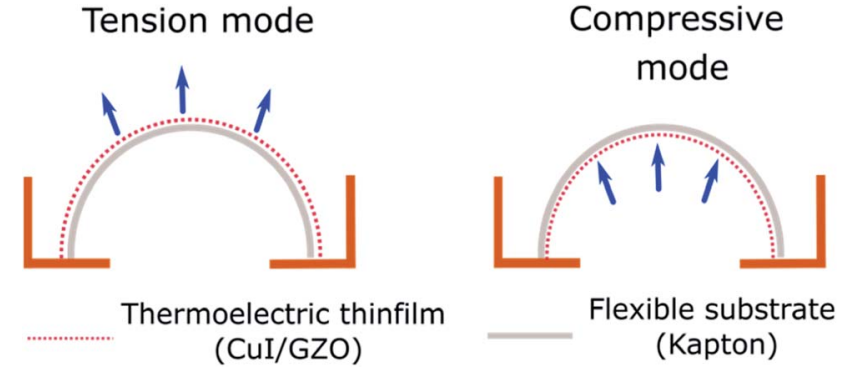

Fig. 3 Tension and compressive forces applied to the thin film on a flexible Kapton substrate during bending analysis.

observed with 100 full bending cycles. This is explained by the imposed compressing forces on the columnar grains of $\mathrm{CuI}$ causing further compacting of the lattice which aids electron flow, whereas the opposite would further disrupt the lattice and ultimately results in film cracks preventing the flow of carriers. Exposing the film to compressive forces after 100 tension cycles results in a reduction of its internal resistance $R_{\text {int }}$ by $7.1 \%$ with a single compressive cycle. A lower $R_{\text {int }}$ value occurs after the $13^{\text {th }}$ compression cycle to yield a resistance value of $0.4 \%$ change from its $R_{0}$ before tension forces were applied; nonetheless, further compression produces a slight increase in $R_{\text {int }}$ after 100 cycles. This is not the case for a film which did not previously undergo tension forces during the bending of the flexible substrate $-R_{\text {int }}$ remains reasonably stable after 100 cycles, as demonstrated in Fig. 5A.

In contrast to the behaviour of CuI, GZO thin films are of ceramic nature with a compact structure and thus showing a resistance variation less sensitive to tension than

Table 1 A summary of thermoelectric properties of Cul and GZO thin films

\begin{tabular}{|c|c|c|c|c|c|c|c|}
\hline Material & $\begin{array}{l}\text { Thickness } \\
(\mathrm{nm})\end{array}$ & $\begin{array}{l}\text { Conductivity } \\
\left(\Omega^{-1} \mathrm{~m}^{-1}\right)\end{array}$ & $\begin{array}{l}\text { Seebeck } \\
\left(\mu \mathrm{V} \mathrm{K} \mathrm{K}^{-1}\right)\end{array}$ & $\begin{array}{l}\text { Power factor } \\
\left(\mathrm{W} \mathrm{m}{ }^{-1} \mathrm{~K}^{-2}\right)\end{array}$ & $\begin{array}{l}\text { Charge carrier density } \\
\left(\times 10^{20} \mathrm{~cm}^{-3}\right)\end{array}$ & $\begin{array}{l}\text { Hall mobility } \\
\left(\mathrm{cm}^{2} \mathrm{~V}^{-1} \mathrm{~s}^{-1}\right)\end{array}$ & $\begin{array}{l}\text { Thermal conductivity } \\
\left(\mathrm{W} \mathrm{\textrm {m } ^ { - 2 }} \mathrm{K}^{-1}\right)\end{array}$ \\
\hline p-type CuI & 302 & $1.1 \times 10^{4}$ & 206.0 & $4.7 \times 10^{-4}$ & 1.7 & 4.1 & 0.48 \\
\hline
\end{tabular}




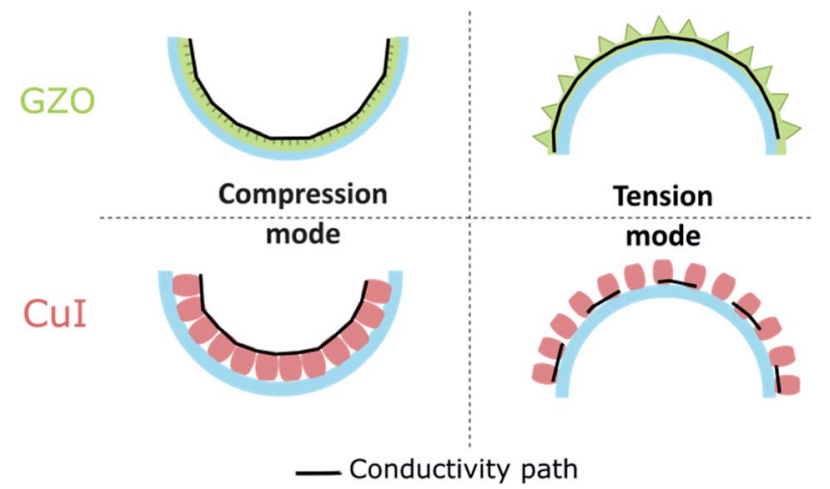

Fig. 4 Conductivity path for GZO and Cul in tension and compression mode.

compression, as shown in Fig. 5C and D. During tension, an increase in resistance of GZO is attributed to the formation of rather superficial cracks in the film, but not deep enough to prevent the flow of carriers (Fig. 6B). However, posterior compression following tensile cycles will result in GZO film cracking in the opposite face of the thin film (in contact with substrate) and thus disturbing the remaining flow of carriers in depth (Fig. 6D). In some cases, GZO thin films which underwent consecutive tensile and compressive cycles revealed areas of an apparent film disintegration or pilling due to excessive cracking even if at a nanoscale.

Overall, the resistance variation over 100 bending cycles is minimal for CuI thin films deposited on transparent Kapton CS, irrespectively of the bending mode used, as it never exceeds $10 \%$. This is a remarkable result.

\subsection{A transparent and flexible thermoelectric generator}

The construction of alternating $\mathrm{p}-\mathrm{n}$ elements connected electrically in series and thermally in parallel increases the application versatility, such refrigeration via the Peltier effect - which requires a $\mathrm{p}-\mathrm{n}$ module - , aside from generally benefitting from two different carrier types to maximise thermoelectric potential at the collection points.

As illustrated in Fig. 7A, indium tin oxide electrodes are deposited at a $6 \mathrm{~mm}$ distance. Power output performances, current-voltage $(I-V)$ and power-voltage $(P-V)$ curves were recorded for pre-defined temperature gradients applied across its planar dimension as opposed to across thickness in bulk materials while varying load resistances. Those temperature gradients were established with a low-diameter $\sim 0.12 \mathrm{~mm}$ wire placed at the heat source located under the substrate and adjacent to the charge injection points (on the heated side) of the $\mathrm{p}-\mathrm{n}$ module. Whilst closing the circuit at both extremities and applying a current, heat is generated in the conductive wire through the Joule heating effect. A fixed temperature gradient is obtained varying the current load. The gradient is measured with the aid of an IR FLIRA310 thermal camera in the front side of the substrate, i.e. the side of the coated TE modules as shown in the thermal map of Fig. $7 \mathrm{~B}$.

In order to correctly assess the efficiency of such transparent and flexible TEG device, several configurations were tested by placing the nanovoltmeter probes at specific locations to access:
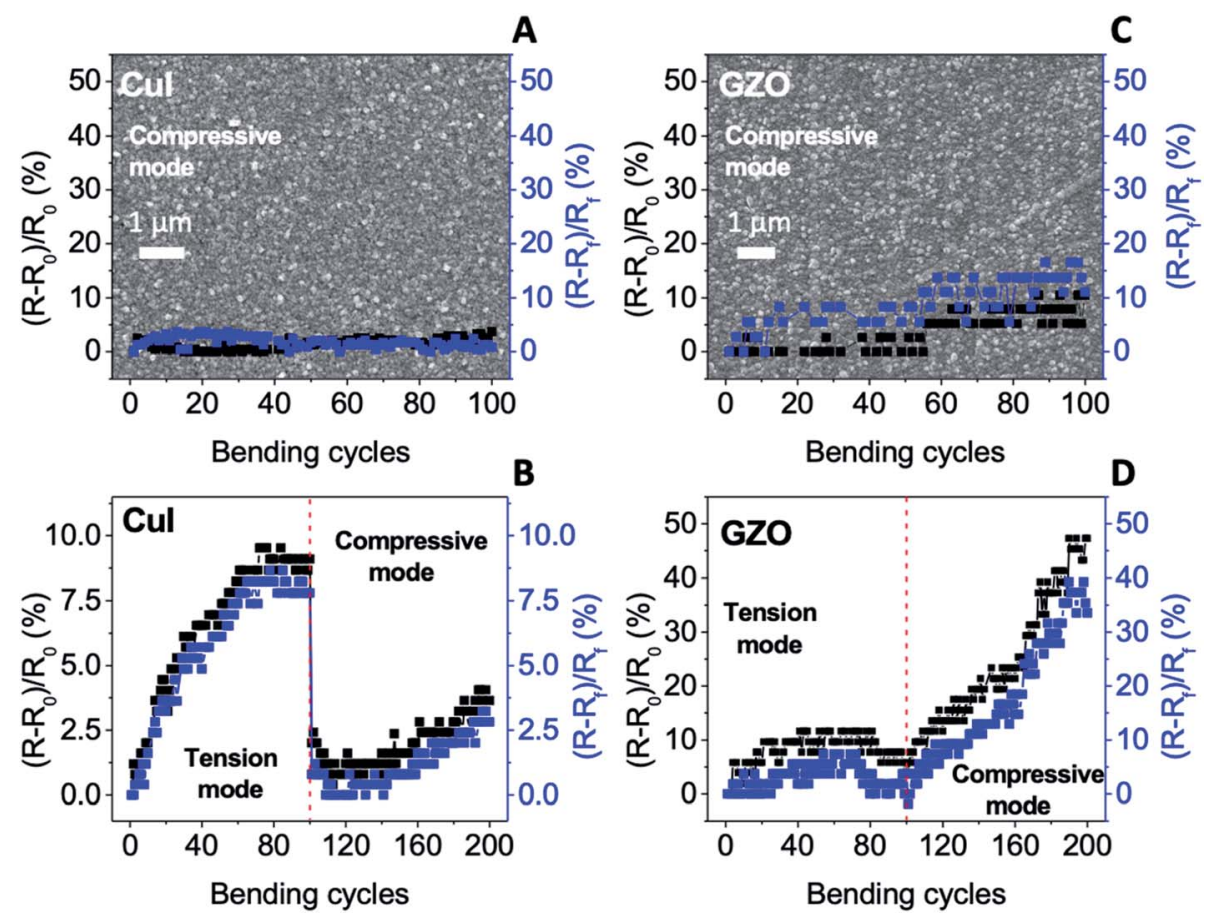

Fig. 5 Bending properties of Cul and GZO thin films deposited on Kapton CS substrate. (A) 100 bending cycles of a single compressive mode of Cul and (C) of GZO (background - corresponding scanning electron micrograph after 100 consecutive cycles in compressive mode). (B) and (D) The effect of 100 cycles in compressive mode after 100 cycles in tension mode. 

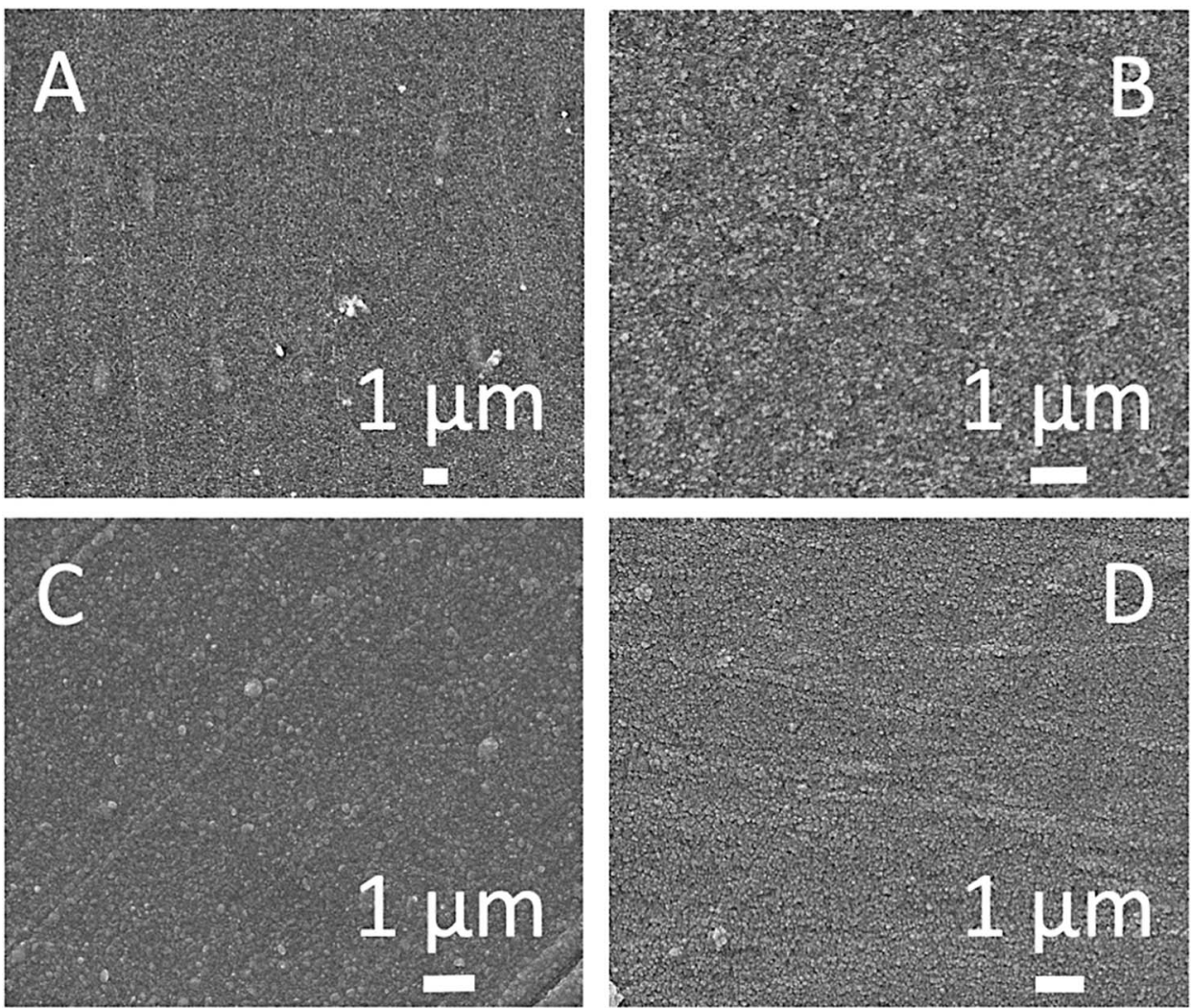

Fig. 6 Scanning electron micrographs of Cul and GZO thin films. (A) Cul thin film on Kapton substrate after 100 tension bending cycles; (B) GZO thin film deposited on Kapton substrate after 100 tension bending cycles; (C) Cul thin film on Kapton substrate after 100 tension cycles plus 100 compression bending cycles; (D) GZO thin film on Kapton substrate after 100 tension cycles plus 100 compression bending cycles.

single, double, triple, quadruple $\mathrm{p}-\mathrm{n}$ modules, and at the total of $17 \mathrm{p}-\mathrm{n}$ modules electrically connected in series. In order to add-up contributions of each TE module, each probe is placed on the electrode at the same temperature (i.e. the potential measured is either by probing at the heat-sink or heated sides of the TEG, but never in opposing configurations).

Current-voltage $(I-V)$ and power-voltage $(P-V)$ characteristic curves were recorded as explained to each of the configurations
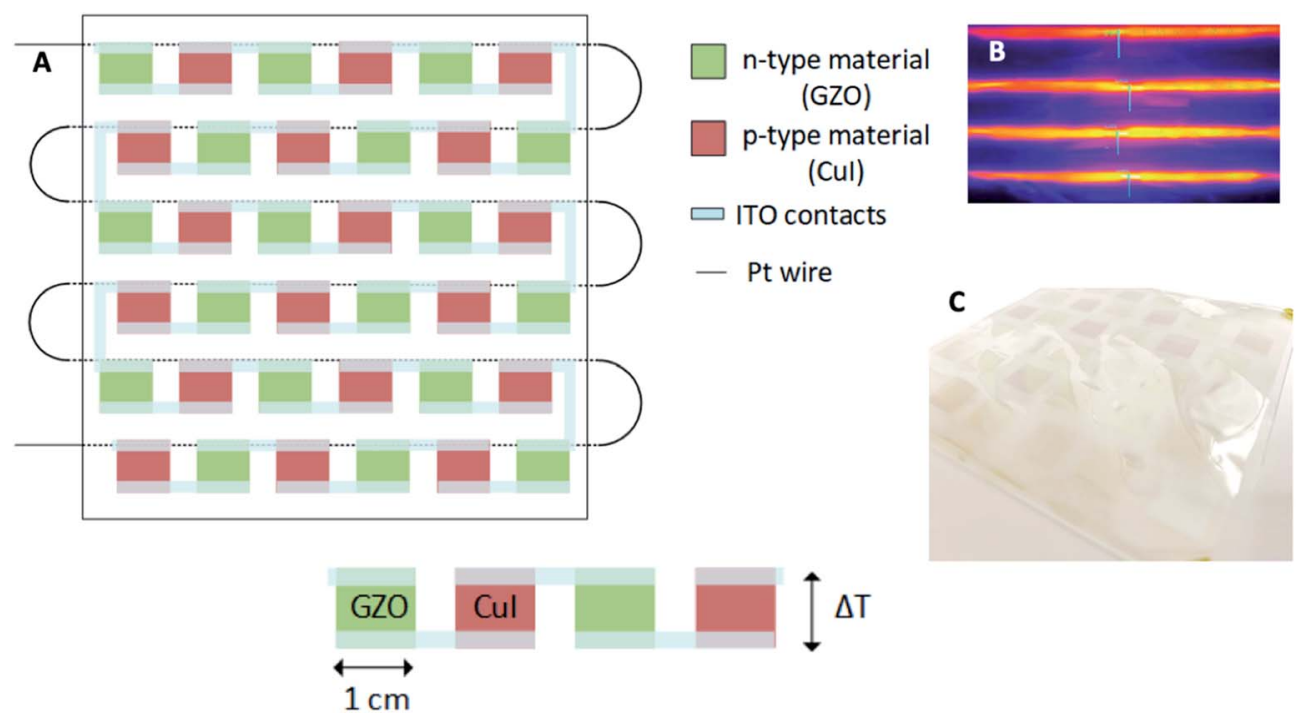

Fig. 7 (A) Illustration of a flexible transparent thermoelectric generator with $17 p-n$ modules. (B) Mapping of the heat dissipation along-side the modules. The Pt wire is heating up the modules, thermally connecting them in parallel. (C) Final Prototype. 
mentioned above. Values of open-circuit voltage $V_{\text {oc }}$, short circuit current $I_{\text {sc }}$ and the maximum output voltage $V_{\text {out }}$ of single elements GZO and CuI increase linearly with increasing $\Delta T$, as described by the generic equation:

$$
V=n\left(S_{\mathrm{p}}-S_{\mathrm{n}}\right) \times \Delta T-I \times R_{\mathrm{int}}
$$

where $V_{\text {out }}$ is the output voltage at the load resistance terminals to obtain the maximum power $\left(P_{\text {out }}\right) ; S_{\mathrm{p}}$ and $S_{\mathrm{n}}$ are respectively the Seebeck coefficient of the $\mathrm{p}$ - and n-semiconductor materials and $n$ is the number of elements connected in series; $I$ is the total current flow in the circuit and $R_{\text {int }}$ the internal resistance of the TE element.

The power output of the device is described by the following equation and adopts a non-linear dependency with $\Delta T$ :

$$
P_{\text {out }}=S \times \Delta T \times I-I^{2} \times R_{\text {int }}
$$

At a $\Delta T$ of $5{ }^{\circ} \mathrm{C}$, a single $1 \times 1 \mathrm{~cm}^{2}$ GZO-CuI p-n module short circuit current $I_{\mathrm{sc}}$ is of $0.29 \mu \mathrm{A}$ and the maximum output voltage $V_{\text {out }}$ is $0.43 \mathrm{mV}$. These values increase linearly with $\Delta T$ as described by the generic equation (eqn (2)) and observed in Fig. 8. Whilst varying load resistance, a maximum power output $\left(P_{\text {out }}\right)$ of $0.51 \mathrm{nW}$ is yielded at a current of $1.16 \mu \mathrm{A}$ for a $\Delta T$ of $20{ }^{\circ} \mathrm{C}$. The increase of power output with temperature follows (eqn (3)).

Although a single $\mathrm{p}-\mathrm{n}$ module may prove insufficient for the supply of low-power applications, multiple $\mathrm{p}-\mathrm{n}$ modules electrically connected in series are certainly capable of reaching the milliwatt range of power density output. As demonstrated in Fig. 8, a total of $17 \mathrm{p}-\mathrm{n}$ modules are able to produce a maximum $P_{\text {out }}$ value of $10.83 \mathrm{nW}$ at $20 \Delta T$ whilst keeping a relatively low $I_{\text {out }}$ value. Nonetheless, interconnection resistance is indisputably a limiting factor on the operation of such prototypes, given the fact that $\mathrm{CuI}$ TE element $R_{\text {int }}$ is already at the lowest conceivable as theoretically predicted ${ }^{6}$ based on the Boltzmann transport theory. As such, lowering $I_{\text {out }}$ values require shortening the distance between ITO interconnections (e.g. by producing in-series-modules along with a more horizontal configuration, as opposed to vertically in series - as these require interconnecting always at the same temperature edge) is expected to influence $I_{\text {out }}$ prominently.

To assess the electrical stability of CuI during heating, a sample of CuI was subjected to increasing temperatures whilst monitoring the change of $R_{\text {int }}$. Firstly, a cycle of increasing and decreasing temperature was applied (Fig. 9A) and later, a continuous increment of $\Delta T$ over time was also experimented (Fig. 9B).

$\mathrm{CuI}$ is stable in air up to $80{ }^{\circ} \mathrm{C}$, beyond which $R_{\text {int }}$ increases significantly and hinders its use in TE applications. A specific cooling time of 15 min was not enough to produce any reversibility in the process, but the rate of change of $R_{\text {int }}$ was lower. Yang et al. ${ }^{9}$ found similar results where $Z T$ values increased up to a temperature of $77{ }^{\circ} \mathrm{C}$, beyond which a decrease in conductivity was similarly observed. However, the authors justify this change in conductivity based on iodide diffusion. Yamada et al., ${ }^{7}$ observed simultaneously a decrease in charge
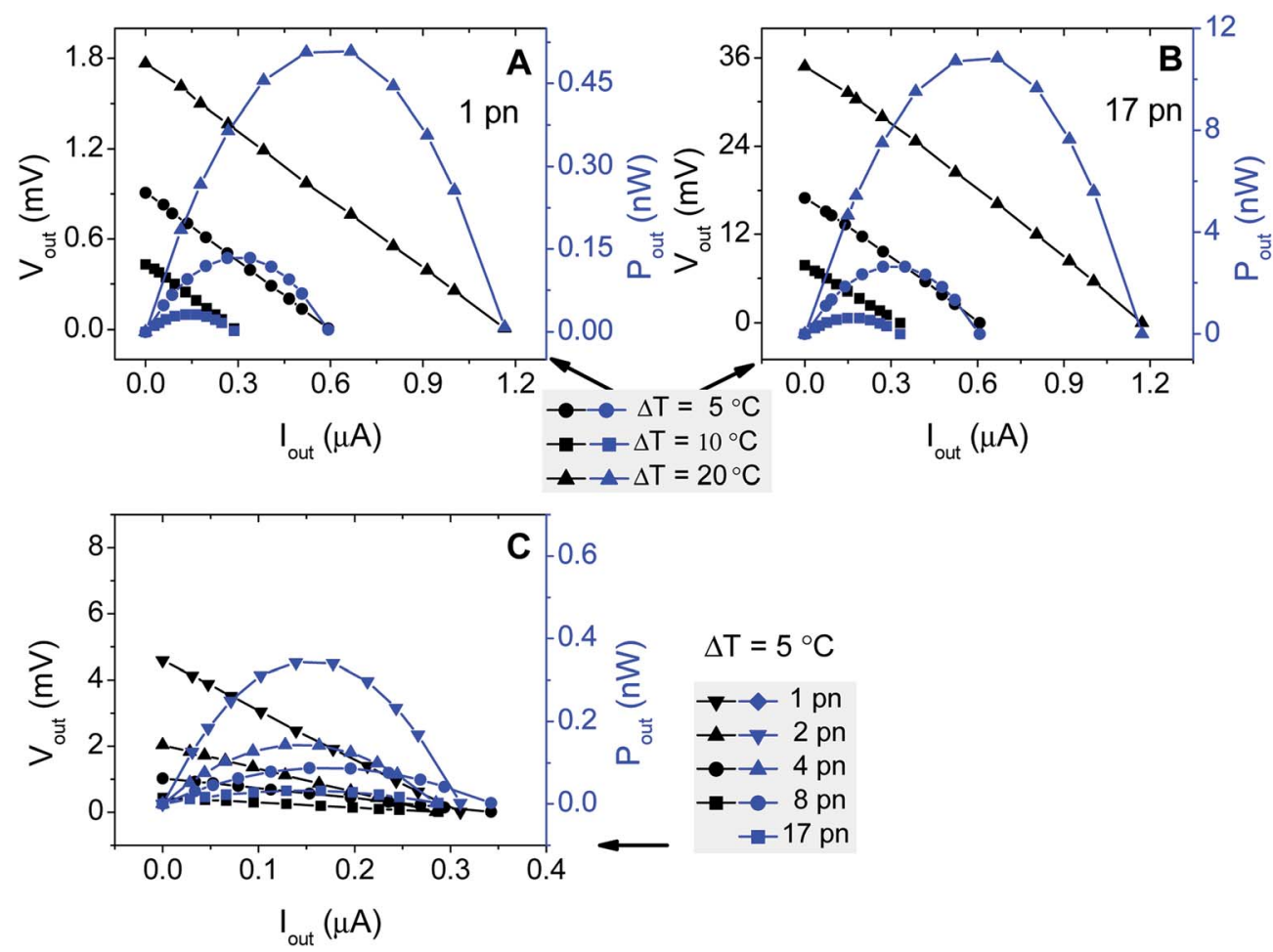

Fig. 8 Current-voltage $(I-V)$ and power-voltage $(P-V)$ characteristic curves varying load resistance and temperature gradients for: (A) $1 \mathrm{p}-\mathrm{n}$ module (Cul/GZO); (B) $17 \mathrm{p}-\mathrm{n}$ modules electrically interconnected with ITO; and (C) All characterized $\mathrm{p}-\mathrm{n}$ modules at a fixed gradient of $5^{\circ} \mathrm{C}$. 

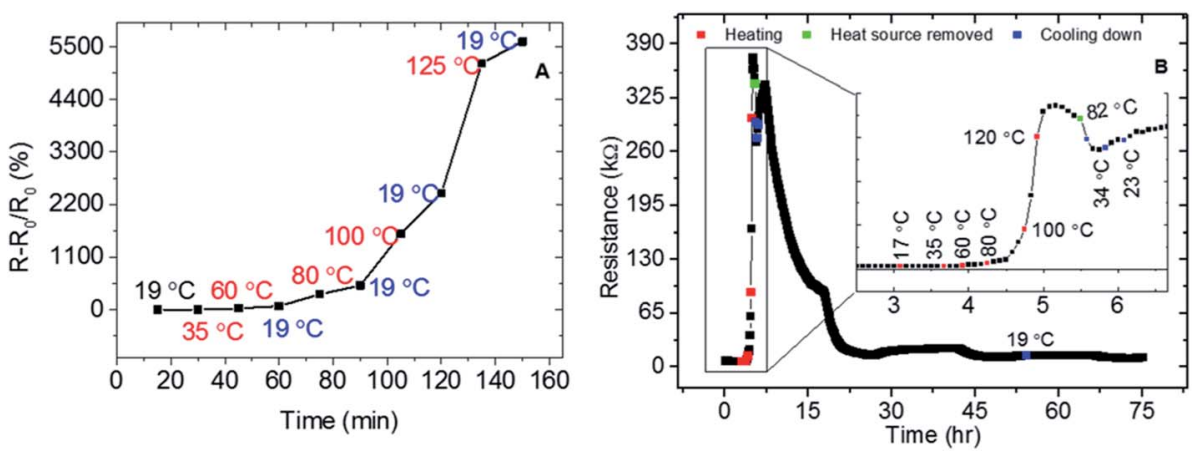

Fig. 9 Change of internal resistance with increasing and decreasing temperature. (A) Cul is exposed to alternating heating a cooling processes at constant rate of heating/cooling; (B) Cul is exposed to constant heating up to $120^{\circ} \mathrm{C}$ and then allowed to cool at room temperature. Different processes of resistance reversibility can be identified.

carrier density $(\eta)$ and an increase in mobility $(\mu)$ with increasing temperature. The authors justify CuI lattices to become stoichiometric as a result of the out-diffusion of copper vacancies during heat treatment. Here we observe the change in resistance to be reversible over a period of $48 \mathrm{~h}$, as shown in Fig. 9B. Whilst copper vacancies may regenerate over time, this reversibility is comparable to the effect of water and hydroxides on oxide semiconductors. ${ }^{12}$

Nevertheless, given the ease of iodide out-diffusion, the possibility of adsorbed oxygen to deplete electron carriers during and post heat treatment in air should not be ruled out. However, the surface adsorption of water molecules is likely to be competing with oxygen and inducing the formation of an electron donor and acceptor area aiding electron conductance at top layers where porosity is greater.

$\mathrm{CuI}$ is a polycrystalline film with high-density grain boundaries and copper vacancies; hence, the uncommon increase in $\mu$ with increasing temperature may only be justified by a decrease in $\eta$ up to a temperature of $100{ }^{\circ} \mathrm{C}$ (Fig. 10). Nonetheless, conductivity decreases significantly and this further evidence the vacancy-related conductivity of the film. Beyond $100{ }^{\circ} \mathrm{C}, \mu$ slightly decreases due to film oxidation and deiodination with relatively constant $\eta$ between $100{ }^{\circ} \mathrm{C}$ and $120^{\circ} \mathrm{C}$, as depicted in Fig. 10.

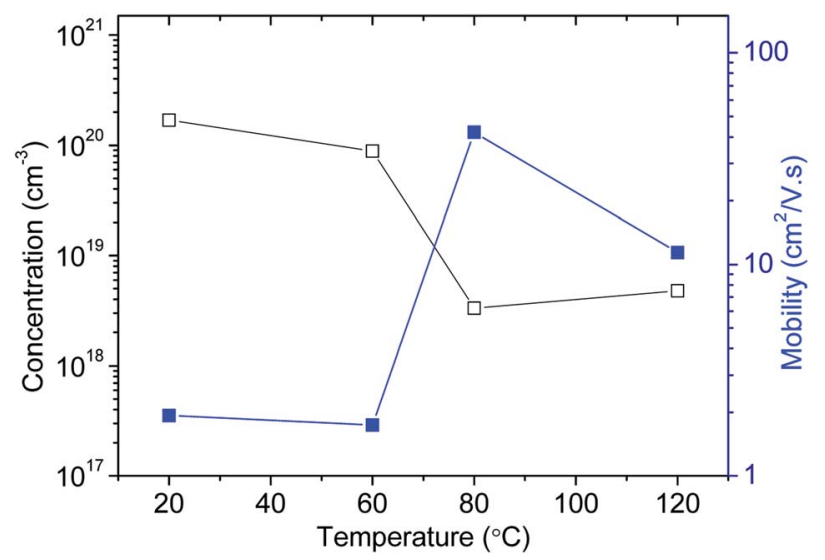

Fig. 10 Concentration and mobility of Cul vs. temperature.

\section{Conclusion}

Thermoelectric p-n modules of CuI-GZO thin films ( $c a .300 \mathrm{~nm})$ were fabricated on a flexible and transparent polyimide Kapton ${ }^{\circledR}$ CS substrate. These were successfully electrically interconnected in series and thermally in parallel to produce a maximum power output of $10.83 \mathrm{nW}$ at a temperature difference of $20^{\circ} \mathrm{C}$ at an $I_{\text {out }}$ of $0.67 \mu \mathrm{A}$.

Additionally, a thin film of copper iodide prepared by solid iodination of a copper thin film demonstrated to behave uniquely to different types of forces applied during bending of the flexible substrate. Under compressive forces, a total of 100 cycles at fixed bending radius results in a relatively stable resistance, whereas under tension forces the $\mathrm{CuI}$ thin film suffers a maximum change of resistance $R_{\text {int }}$ of $9.1 \%$ after 100 bending cycles. Furthermore, the resistance recovery of a sample subjected to tension is possible when a compressive force is subsequently applied.

Moreover, $\mathrm{CuI}$ thin films were observed to be electrically unstable at temperatures beyond $80{ }^{\circ} \mathrm{C}$ as this stoichiometrically homogenises the film and eliminates vacancy-related electron conductance. Nevertheless, the process is mostly reversible over time, and $R_{\text {int }}$ recovery is achievable with the adsorption of hydroxide molecules at the surface of the thin film - a process commonly observed in oxide semiconductors.

The results presented in here are key to define the potential future applications of $\mathrm{CuI}$ thin films in transparent technology, not only in the field of thermoelectrics but on a broader scale of applications requiring simultaneously transparent, flexible, electrically conductive p-type materials.

\section{Conflicts of interest}

There are no conflicts to declare.

\section{Acknowledgements}

This work was mainly funded by H2020-ICT-2014-1, RIA, TransFlexTeg-645241, and ERC-CoG-2014, CapTherPV, 647596, and partially funded by FEDER funds through the COMPETE 2020 Program and National Funds through FCT - Portuguese 
Foundation for Science and Technology under the project UID/ CTM/50025/2019.The authors would like to thank David Sousa and Ana Sofia Taborda for writing the software used for controlling the bending machine and measuring the resistance of the AZO films, and also to Doctor R. C. da Silva for developing the post-processing software, required for determining the relevant parameters per cycle: e.g. bending radius of curvature, initial and final electrical resistances as well as the critical bending radius.

\section{References}

1 M. Grundmann, F. Klüpfel, R. Karsthof, P. Schlupp, F.-L. Schein, D. Splith, C. Yang, S. Bitter and H. von Wenckstern, J. Phys. D: Appl. Phys., 2016, 49, 213001.

2 H. Kawazoe, M. Yasukawa, H. Hyodo, M. Kurita, H. Yanagi and H. Hosono, Nature, 1997, 389, 939.

3 C. Ruttanapun, W. Kosalwat, C. Rudradawong, P. Jindajitawat, P. Buranasiri, D. Naenkieng, N. Boonyopakorn, A. Harnwunggmoung, W. Thowladda, W. Neeyakorn, C. Thanachayanont, A. Charoenphakdee and A. Wichainchai, Energy Procedia, 2014, 56, 65.
4 K. Hayashi, K. I. Sato, T. Nozaki and T. Kajitani, Jpn. J. Appl. Phys., 2008, 47, 59.

5 B. M. Morais Faustino, D. Gomes, J. Faria, T. Juntunen, G. Gaspar, C. Bianchi, A. Almeida, A. Marques, I. Tittonen and I. Ferreira, Sci. Rep., 2018, 8, 6867.

6 M. K. Yadav and B. Sanyal, Mater. Res. Express, 2014, 1, 015708.

7 N. Yamada, R. Ino, H. Tomura, Y. Kondo and Y. Ninomiya, Adv. Electron. Mater., 2017, 3, 1700298.

8 S. Qing, A. Rezania, L. A. Rosendahl and X. Gou, Mater. Today: Proc., 2018, 5, 10338.

9 C. Yang, D. Souchay, M. Kneiß, M. Bogner, H. M. Wei, M. Lorenz, O. Oeckler, G. Benstetter, Y. Q. Fu and M. Grundmann, Nat. Commun., 2017, 8, 16076.

10 S.-I. Park, J.-H. Ahn, X. Feng, S. Wang, Y. Huang and J. A. Rogers, Adv. Funct. Mater., 2008, 18, 2673.

11 M. Grundmann, F.-L. Schein, M. Lorenz, T. Böntgen, J. Lenzner and H. von Wenckstern, Phys. Status Solidi, 2013, 210, 1671.

12 J.-S. Park, J. K. Jeong, H.-J. Chung, Y.-G. Mo and H. D. Kim, Appl. Phys. Lett., 2008, 92, 72104. 